\title{
Producción e impacto de las revistas peruanas del ámbito de las Ciencias Sociales en el catálogo Latindex
}

\author{
Luís Fernando Morales Morante*
}

Artículo recibido:

7 de agosto de 2014.

Artículo aceptado:

14 de mayo de 2015.

\section{Resumen}

En el presente artículo se analiza la producción científica y los principales indicadores de visibilidad internacional de 25 revistas peruanas del campo de las Ciencias Sociales indexadas en el Catálogo Latindex entre los años 2005-2013. Los resultados indican que hay un mayor número de revistas del campo de la Psicología, Economía y Derecho respecto del resto de áreas de conocimiento. Se observa un volumen importante de artículos publicados así como un equilibrio entre artículos a cargo de autores nacionales y extranjeros. Se constata un claro dominio de las universidades privadas frente a las públicas en la tarea editorial de revistas científicas. Se proponen acciones e iniciativas de incen-

\footnotetext{
Universidad Autónoma de Barcelona, Barcelona, España. fernando.morales@uab.es

INVESTIGACIÓN BIBLIOTECOLÓGICA, Vol.30, Núm.69, mayo/agosto, 2016, México, ISSN: 0187-358X. pp. 179-204
} 
tivo para mejorar la calidad, número de citas y la presencia en motores de búsqueda internacional.

Palabras clave: Latindex; Perú; Visibilidad; Impacto; Ciencias Sociales.

\section{Abstract}

Production and impact of Peruvian social science journals in the Latindex catalogue

Luís Fernando Morales-Morante

This paper analyzes the scientific production and leading indicators of international visibility of 25 Peruvian social science journals indexed in Latindex between 2005 and 2013, finding, with respect to other areas of knowledge, that there are more journals in the field of Psychology, Economy and Law and a large, relatively balanced quantity of articles published by both national and foreign authors. Moreover, papers by authors associated with private universities appear more often in the pages of these journals than those from public universities. Some incentive measures are proposed to improve the quality, number of citations and presence in international search engines.

Keywords: Latindex; Peru; Visibility; Impact; Social Science.

\section{INTRODUCCIÓN}

L as cifras internacionales reflejan un importante crecimiento económico en algunos países de la región de América del Sur en los últimos años. Por sus indicadores macroeconómicos, Perú es uno de los países que recientemente es utilizado como paradigma de dicho crecimiento con una tasa promedio superior al $5 \%$ en los últimos cinco años. En el campo educativo, este crecimiento se ha visto reflejado en la creación de un número importante de universidades privadas y algunas públicas. Una manera objetiva de comprobar si este auge expansivo es consistente es a través del análisis de la producción científica de sus revistas. Es útil en la medida que nos permite constatar si detrás de la oferta universitaria existe una verdadera producción 
intelectual que la respalde, si hay redes de investigadores a su alrededor que elaboran conjuntamente esa literatura, si se cumple con unos niveles de calidad y si esa producción es visible en el contexto nacional e internacional. También puede ayudarnos a detectar las fortalezas y debilidades de las políticas actuales y visualizar líneas de acción futuras para corregirlas y mejorarlas. Confrontar el flujo de dichos datos en las revistas del ámbito de las Ciencias Sociales resulta especialmente importante, porque es precisamente en esta área de conocimiento donde se ubica el mayor número de carreras y facultades del país (Derecho, Comunicaciones, Economía, Psicología) y se evidencia a la par un incremento considerable en el volumen de revistas científicas indexadas en el catálogo Latindex.

Las pruebas indican que no existen investigaciones bibliométricas que se hayan ocupado en analizar la evolución o el cumplimiento de al menos algunos de sus parámetros de calidad. Creemos que un estudio exploratorio e inicial de estas características ayuda a disponer de una representación general de cómo se organiza la producción científica en esta área de conocimiento y cuáles son algunos de sus estándares de calidad objetivos actuales.

\section{MarCo TEÓRICO}

\section{Estudios bibliométricos en el ámbito de las ciencias médicas en el Perú}

Desde finales de la década de los noventa comenzó a desarrollarse una línea de investigación bibliotecológica centrada en analizar los parámetros de calidad y visibilidad de las revistas editadas en el Perú, concretamente en el campo de la medicina y las biociencias. Huamaní y Pacheco examinan, por ejemplo, los indicadores de la producción científica colaborativa en el campo médico (Huamaní y Pacheco-Romero, 2011; Huamaní y Mayta-Tristán, 2010). Otros estudios analizan las deficiencias y el ajuste de las políticas editoriales dirigidas para mejorar el posicionamiento internacional de las publicaciones (Burstein y Cabezas, 2010; Huamaní y Pacheco-Romero, 2009; Arroyo-Hernández, Zukerán-Medina y Miranda-Soberón, 2009). Romaní y Wong (2009) examinan los protocolos de filtraje para la búsqueda de artículos en al catálogo Scielo, así como sus principales indicadores de visibilidad. Cáceres y Mendoza (2009) proponen un análisis global de la producción científica en el campo médico. Las conclusiones principales constatan un bajo nivel de producción e indización de esta clase de publicaciones, pese a ello es la más alta en comparación con otros ámbitos (Huamaní y Pacheco-Romero, 2011; Romaní y Wong, 2009; Gerstein, 2005). Como posibles causas 
de la poca productividad se detectaron los procesos rigurosos y largos que determinan la publicación de los artículos (Burstein y Cabezas, 2010), así como la distribución de los originales y la selección de expertos para el proceso de revisión por pares (Cuevas y Mestaza, 2002). Un dato positivo es que se puede constatar una elevada visibilidad electrónica a través de motores de búsqueda por Internet (Huamaní y Pacheco-Romero, 2009).

Otra vía de trabajo ciertamente menos prolífica que la anterior explora el aporte de los estudiantes en la publicación de artículos científicos en revistas médicas (Taype-Rondán et al., 2011; Alarcón-Villaverde, Romaní y Gutiérrez, 2010; Mayta-Tristán y Pena-Oscuvilca, 2009; Molina-Ordóñez, Huamaní y Mayta-Tristán, 2008; Huamaní, Chávez-Solís y Mayta-Tristán, 2008; Gutiérrez y Mayta-Tristán, 2003). Los resultados indican que son evidentes los esfuerzos de las instituciones educativas y centros de investigación peruanos por publicar y pertenecer a sistemas reconocidos de indexación. Dicha labor aporta un valor añadido a las instituciones para exhibir su calidad, mejorar su visibilidad en el contexto internacional e hilvanar nuevos mecanismos de interrelación con grupos de investigación que en el futuro lleven a la producción de nuevos artículos.

\section{Ámbito de las Ciencias Sociales en España como paradigma latinoamericano}

En la década pasada España ha comenzado a experimentar un proceso de modificación de los criterios para el acceso al cuerpo de profesores universitarios funcionario y contratado. Para ello los sistemas de examen para obtener la habilitación y acreditación correspondientes se han visto modificados con la incorporación de méritos objetivos internacionales para la valoración de la actividad investigadora. Esto ha hecho que se desarrollen modelos de referencia internacional, aplicando criterios similares a los usados por los grandes sistemas de clasificación de revistas como ISI-JCR (Thomson Reuters) o Scopus. En las Ciencias Sociales se dispone así de diferentes herramientas para medir la calidad de las revistas (impact factor, factor de impacto) a partir de la indización sistemática de las referencias bibliográficas citadas así como otros criterios representativos. IN-RECS ${ }^{1}$ calcula el impacto de 761 revistas de Ciencias Sociales organizadas en diez ámbitos. La base de consulta de acceso libre a la fecha ha tenido más de 878507 visitas registradas. DICE: Difusión y Calidad Editorial de las Revistas Españolas de Humanidades y Ciencias Sociales y Jurídicas surge fruto de un convenio de colaboración entre el Consejo Superior de Investigaciones Científicas (CSIC) 
y la Agencia Nacional de Evaluación de la Calidad y Acreditación (ANECA). Proporciona dos indicadores, valor de difusión internacional y nivel de internacionalidad, a partir de la cuantificación y valor de las citaciones, junto con otros datos relevantes para investigadores y editores. La búsqueda puede efectuarse por revista o por el conjunto de las revistas del área. MIAR ${ }^{2}$ es un sistema para medir cuantitativamente la visibilidad de las publicaciones periódicas en Ciencias Sociales en función de su presencia en distintos tipos de bases de datos.

Existen varias herramientas complementarias que no suministran específicamente índices de impacto o de visibilidad de las publicaciones pero que ofrecen otros indicadores relevantes asociados con la calidad de las publicaciones. La plataforma Resh ${ }^{3}$ sistematiza los criterios cumplidos por las revistas según los parámetros de calidad fijados por tres instituciones evaluadoras: CNEAI, ANECA y Latindex. Se trata de proveer indicadores de calidad editorial, un sistema de evaluación de originales, apertura institucional de los órganos de gestión editorial, internacionalidad, entre otros. Otros instrumentos son también utilizados en Ciencias Sociales cuando no se dispone de información cuantitativa específica. En este grupo tenemos sistemas basados en la opinión de expertos que determina la calidad en 3 niveles de clasificación, como en el sistema catalán Cahrus+. ${ }^{4}$

No obstante, desde una perspectiva latinoamericana son evidentes las dificultades para confeccionar un sistema eficiente que conjugue estos y otros indicadores de manera consensuada y accesible desde diferentes fuentes y bases de datos, y aglutine toda la variedad de publicaciones existentes. En términos generales y ante dicha imposibilidad, la búsqueda de revistas y artículos se efectúa únicamente mediante tres grandes motores: Redalyc (México), Scielo y Dialnet.

En el Perú existe el motor Scielo-Perú, coordinado por el Consejo Nacional de Ciencia, Tecnología e Innovación Tecnológica (CONCYTEC), la Universidad Nacional Mayor de San Marcos y la Representación en Perú de la Organización Panamericana de la Salud. En el campo específico de Humanidades se registran cinco revistas de Ciencias Sociales y un total de 38 volúmenes disponibles:

1. Antbropologica, 9 números

2. Areté. Revista de Filosofía, 13 números

\footnotetext{
http://miar.ub.edu/es [Fecha de consulta: 25/10/2014]

http://epuc.cchs.csic.es/resh/que_es [Fecha de consulta: 25/10/2014]

4 http://www10.gencat.cat/agaur_web/AppJava/castellano/a_info.jsp?contingut=carhus_2014 [Fecha de consulta: 25/10/2014]
} 
3. Journal of Economics, Finance and Administrative Science, 8 números

4. Liberabit. Revista de Psicología, 13 números

5. Revista de Psicología (PUCP), 6 números

Diferentes estudios se han efectuado para complementar la funcionalidad de estos procedimientos y facilitar la obtención de resultados en las revistas científicas de Ciencias Sociales, y así obtener otros indicadores comparativos que los sistemas actuales no son capaces de suministrar a los investigadores. López Baena, Valcárcel Cases y Barbancho Medina (2005) efectúan un protocolo de evaluación de publicaciones como complemento de la base de datos ISI. En otra vía se exponen los protocolos bibliométricos del catálogo Resh (Alcain, Román Román y Giménez Toledo, 2008) y Latindex (Alcain y Lascurain, 2002). Ambos sustentan la calidad del proceso editorial, la revisión por pares, la visibilidad internacional y los índices de impacto, entre otros indicadores. Borrego y Urbano (2006) examinan las dificultades de aplicación de los métodos bibliométricos de repercusión basados en el análisis de citas con datos obtenidos de las bases de datos de Thomson Scientific (ex ISI). Vázquez, Urdín Caminos y Román Román (2003) examinan el comportamiento de las revistas españolas de ciencias de la salud frente a los criterios de calidad editorial Latindex y su grado de accesibilidad por Internet. En el campo específico de la Comunicación, los trabajos de Fernández Quijada (2010, 2009) analizan las similitudes y diferencias entre las diferentes revistas. Román Román, Vázquez Valero y Urdín Camino (2002) analizan los criterios de calidad editorial Latindex en el marco de la evaluación de las revistas españolas de Humanidades y Ciencias Sociales. Alcain y Lascurain (2002) analizan las 221 revistas de Psicología latinoamericanas. Gamboa (1998) efectúa un prototipo de índice latinoamericano de publicaciones científicas Latindex. Sin embargo, en el campo específico de las Ciencias Sociales, no existen estudios que perfilen la producción de los autores e instituciones y que permita efectuar comparaciones y valorar el impacto según indicadores objetivos validados.

\section{Oвjetivos}

El presente artículo se propone los tres objetivos siguientes:

1. Efectuar un estudio cuantitativo y cualitativo de la producción científica de las revistas peruanas del ámbito de las Ciencias Sociales, presentes en el catálogo Latindex entre los años 2005 y 2013, a partir de indicadores estandarizados de impacto. 
2. Perfilar de acuerdo a los resultados del análisis de datos un esquema del nivel de posicionamiento de las revistas e instituciones que las producen ante la comunidad científica internacional.

3. Proponer medidas orientadas a mejorar la calidad y el nivel de impacto internacional de las publicaciones.

\section{Metodología}

El análisis aplicado en este estudio se basa en un recuento bibliométrico de los artículos publicados en revistas científicas entre el año 2005 y 2013. Se escogió dicho periodo porque marca un intervalo amplio, suficiente para poder advertir la evolución de las publicaciones y obtener, finalmente, una perspectiva longitudinal de la investigación más reciente en este ámbito, concordante con el periodo de crecimiento de la oferta universitaria nacional.

El proceso de investigación documental se llevó a cabo en cuatro fases:

1. Localización de la totalidad de revistas indexadas publicadas en Perú del ámbito de Ciencias Sociales en el catálogo Latindex. Para efectuarla se ingresa al buscador avanzado de Latindex/Tema: Ciencias Sociales/Indizada-Resumida en: Latindex Catálogo/País: Perú.

2. Inventario de la totalidad de artículos publicados por cada revista entre los años 2005-2013. La recolección de datos se realiza desde la página web oficial de cada una de las publicaciones o mediante motores de búsqueda especializados como Scielo, Amauta o Redalyc.

3. Construcción de una base de datos de los artículos científicos publicados por las revistas conteniendo trece variables relevantes.

4. Análisis comparativo de los indicadores durante el periodo de análisis estipulado, organizado en tres campos y seis categorías.

\section{Indicadores del análisis}

La elección de las variables de análisis se realiza con el objetivo de obtener información de las publicaciones en tres campos (Tabla 1):

1. Datos generales. Indican el número de revistas, especialidad temática, institución que la edita y frecuencia de la publicación.

2. Producción científica. Este campo persigue cuantificar la producción científica de cada revista y además conocer cómo se organiza la autoría de los artículos. 
3. Indicadores de calidad. Incluye la visibilidad de la revista en otros motores, número de citaciones en Google Académico (GA), Clase y número de criterios Latindex cumplidos.

Tabla 1. Indicadores de análisis

\begin{tabular}{|c|c|c|c|}
\hline Campos & & Variables & Descriptor \\
\hline \multirow[t]{5}{*}{ Datos generales } & 1 & Número de revistas & \multirow{5}{*}{$\begin{array}{l}\text { Indica la distribución de la producción editorial de las } \\
\text { revistas según especialidades, centros y periodicidad } \\
\text { de publicación }\end{array}$} \\
\hline & 2 & Área de especialidad & \\
\hline & 3 & Institución & \\
\hline & 4 & Naturaleza de la institución & \\
\hline & 5 & Frecuencia de la publicación & \\
\hline \multirow{5}{*}{$\begin{array}{l}\text { Producción } \\
\text { científica }\end{array}$} & 1 & Artículos publicados & \multirow{5}{*}{$\begin{array}{l}\text { Muestra el volumen de artículos, la distribución de au- } \\
\text { tores e internacionalidad de las contribuciones }\end{array}$} \\
\hline & 2 & Autores nacionales & \\
\hline & 3 & Autores extranjeros & \\
\hline & 4 & Autoría múltiple & \\
\hline & 5 & Int. de las contribuciones & \\
\hline \multirow{3}{*}{$\begin{array}{l}\text { Indicadores } \\
\text { de calidad }\end{array}$} & 1 & Indexación en otros motores & \multirow{3}{*}{$\begin{array}{l}\text { Valora la calidad a partir de la indexación en otros mo- } \\
\text { tores, citaciones y criterios Latindex cumplidos }\end{array}$} \\
\hline & 2 & Número de citaciones GA & \\
\hline & 3 & Criterios Latindex cumplidos & \\
\hline
\end{tabular}

\section{Resultados}

El resultado del buscador muestra un total de 45 títulos de revistas, que sin discriminación inicial se analizan individualmente y cuyos datos se ingresan en un fichero especial en formato XLS para poder construir tablas estadísticas básicas de sus resultados. En una primera revisión constatamos que en dicho listado aparece extrañamente la revista de Ingemmet, una publicación del campo de la Ingeniería; por no corresponder al ámbito de este estudio queda descartada. Observamos también que 10 revistas no están disponibles en la Web, por tanto, no podemos disponer de información para incorporar al análisis. Una vez concluido el levantamiento de datos de cada revista, comprobamos que algunas han comenzado a publicarse varios años después del 2005, otras han dejado de circular antes del 2013 y otras tienen intervalos prolongados de ausencia (Anexo 1). Si decidiéramos incluirlas podrían dar una visión incorrecta de la productividad en comparación con las publicaciones que sí han sido regulares en el periodo fijado, por tanto, nos vimos ante la necesidad de fijar un criterio de inclusión en el análisis. Limitamos un máximo de tres números anuales, porque dicho intervalo de no presencia es aceptable tomando en cuenta que el periodo analizado es de 8 años. Es razonable para que no afecte la lectura comparativa del conjunto y porque dicho lapso 
puede contemplar situaciones que expliquen una interrupción involuntaria o simplemente un retraso sólo de la publicación en versión electrónica. Luego del filtraje se procede a efectuar el análisis comparativo de 25 revistas, con los resultados siguientes (Tabla 2 ).

\section{Campo 1. Datos generales}

Número de revistas

\section{5}

\section{Areas de especialidad}

Visualizando el Gráfico 1, apreciamos que la mayor concentración se produce en el área de Psicología, con un total de 6 revistas indexadas. Le siguen ADE-Economía y Arqueología, Antropología e Historia con un total de 5, luego Derecho con 4. Más relegadas vemos Comunicación con 2 y con una sola publicación 3 revistas: una de Biblioteconomía, otra de Educación y finalmente una de Sociología.

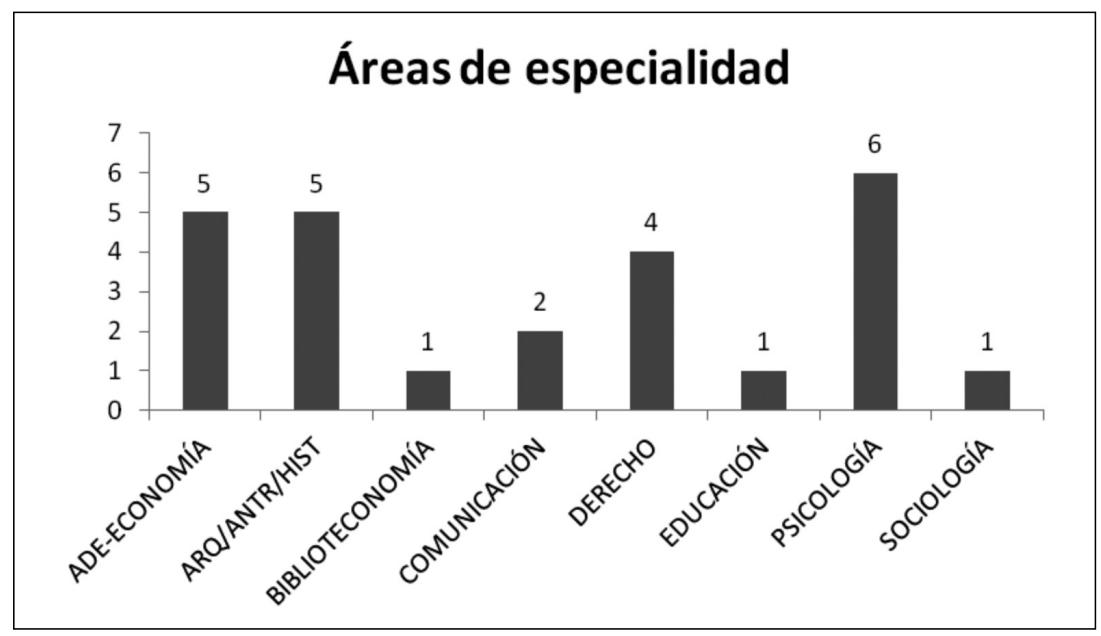

Gráfico 1. Distribución por áreas

\section{Institución}

Aquí podemos observar la producción editorial por instituciones (Gráfico 2). Notamos que el centro con mayor número de revistas indexadas es la Pontifi- 
cia Universidad Católica del Perú con 9 revistas, luego la Universidad Nacional Mayor de San Marcos con 3 publicaciones. Con 2 la Universidad de Lima y la Universidad de Piura, también privadas. El resto de centros sólo edita una publicación: Biblios, Esan, Instituto de Estudios Amazónicos, Instituto Nacional de Defensa de la Competencia y de la Protección de la Propiedad Intelectual, Onpe, Universidad César Vallejo, Universidad Femenina, Universidad del Pacífico y Universidad San Martín de Porres.

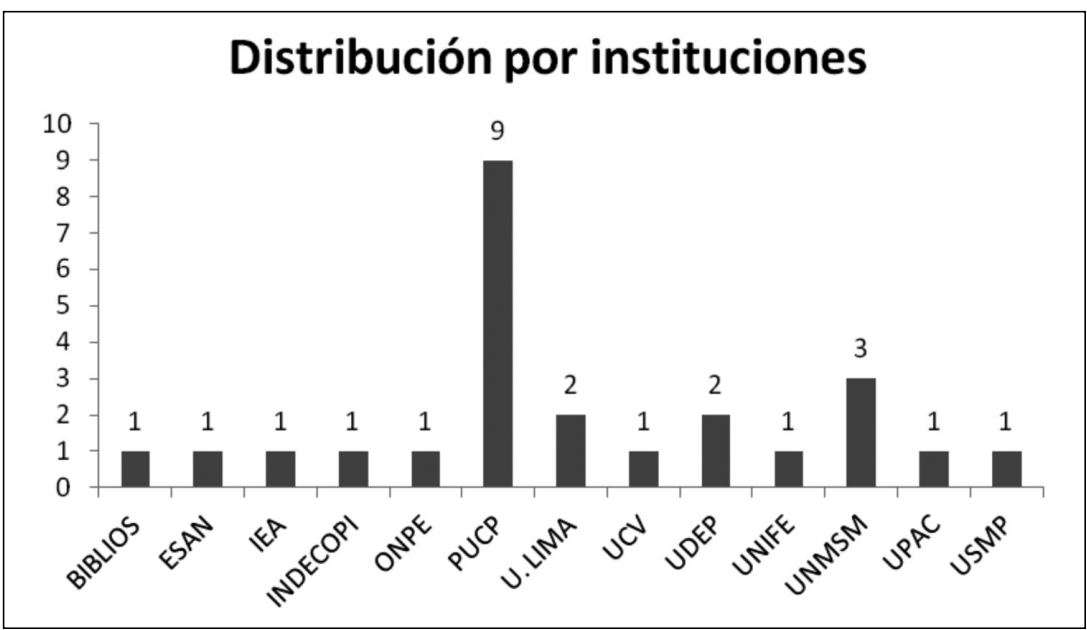

Gráfico 2. Distribución por instituciones

Del total de publicaciones (Gráfico 3, pág. s.) 18 corresponden a universidades privadas, 3 a la universidad pública Mayor de San Marcos. Dos a centros privados no universitarios (editorial Biblios y el Instituto de Estudios Amazónicos), que editan revistas con el mismo nombre. Finalmente, las dos restantes corresponden a intituciones públicas descentralizadas: la Oficina Nacional de Procesos Electorales (Onpe) que edita la revista Elecciones y el Instituto Nacional de Defensa de la Competencia y de la Protección de la Propiedad Intelectual (Indecopi), que edita la Revista de la Competencia y la Propiedad Intelectual.

\section{Frecuencia de la publicación}

Se publican anualmente 14 revistas, mientras que 11 lo hacen con una periodicidad de dos números por año.

En la Tabla 2 (pág. s.) podemos visualizar el desglose individual de este primer apartado del análisis. 


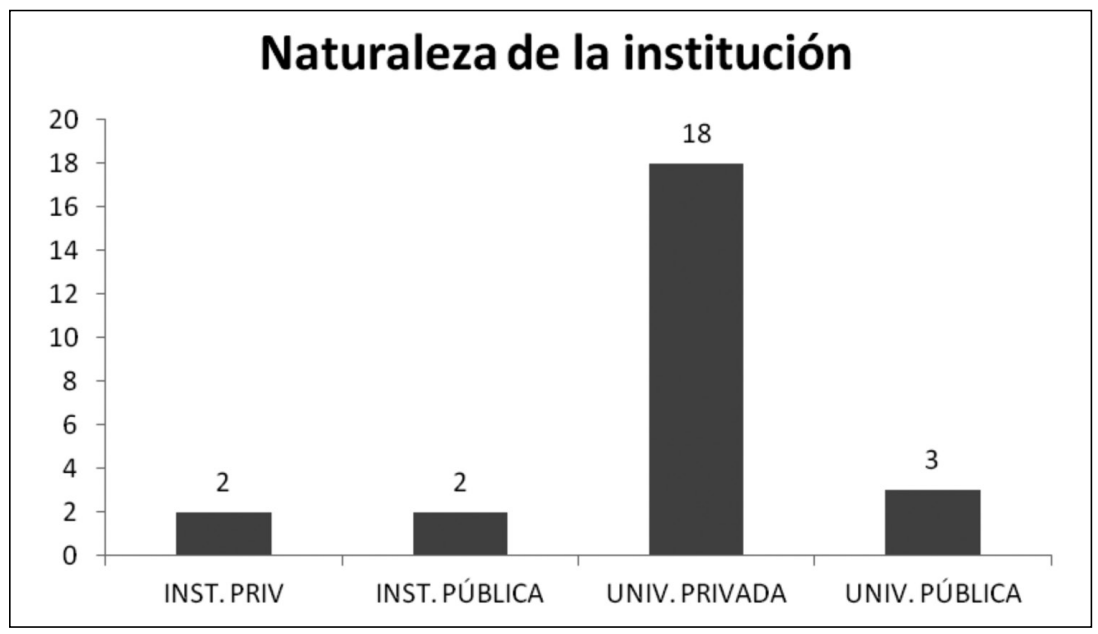

Gráfico 3. Naturaleza de la institución

Tabla 2. Datos generales

\begin{tabular}{|c|c|c|c|c|c|}
\hline & Revista & Área & Institución & Nat. Inst. & Frecuencia \\
\hline 1 & Anthropologica & Arq/Antr/Hist & U. Católica & U. Privada & Anual \\
\hline 2 & Apuntes & ADE-Economía & U. Pacífico & U. Privada & Semestral \\
\hline 3 & Arqueología y Sociedad & Arq/Antr/Hist & U. San Marcos & U. Publica & Anual \\
\hline 4 & Avances en Psicología & Psicología & U. Femenina & U. Privada & Anual \\
\hline 5 & Biblios & Biblioteconomia & Grupo Biblios & Inst. Priv & Semestral \\
\hline 6 & Boletín de Arqueología & Arq/Antr/Hist & U. Católica & U. Privada & Anual \\
\hline 7 & Contabilidad y Negocios & ADE-Economía & U. Católica & U. Privada & Semestral \\
\hline 8 & Contratexto & Comunicación & U. de Lima & U. Privada & Anual \\
\hline 9 & CD J.of Economics & ADE-Economía & E. Negocios & U. Privada & Semestral \\
\hline 10 & Debates de Sociología & Sociología & U. Católica & U. Privada & Anual \\
\hline 11 & Derecho PUCP & Derecho & U. Católica & U. Privada & Anual \\
\hline 12 & Economía & ADE-Economía & U. Católica & U. Privada & Anual \\
\hline 13 & Elecciones & Derecho & ONPE & Inst. Púb. & Anual \\
\hline 14 & Estudios Amazónicos & Arq/Antr/Hist & IEA & Inst. Priv. & Anual \\
\hline 15 & Histórica & Arq/Antr/Hist & U. Católica & U. Privada & Semestral \\
\hline 16 & Investigación Educativa & Educación & U. San Marcos & U. Publica & Semestral \\
\hline 17 & J. of CENTRUM Cathedra & ADE-Economía & U. Católica & U. Privada & Semestral \\
\hline 18 & Liberabit & Psicología & U. San Martín & U. Privada & Semestral \\
\hline 19 & Persona & Psicología & U. de Lima & U. Privada & Anual \\
\hline 20 & Comunicación & Comunicación & U. de Piura & U. Privada & Anual \\
\hline 21 & Revista de Derecho & Derecho & U. de Piura & U. Privada & Anual \\
\hline 22 & Revista de la Prop. Int. & Derecho & INDECOPI & Inst. Púb. & Anual \\
\hline 23 & Inv. en Psicología & Psicología & U. San Marcos & U. Publica & Semestral \\
\hline 24 & Revista de Psic. PUCP & Psicología & U. Católica & U. Privada & Anual \\
\hline 25 & Revista de Psic. UCV & Psicología & U. C. Vallejo & U. Privada & Semestral \\
\hline
\end{tabular}




\section{Campo 2. Producción cientifica}

En total se registraron 2475 artículos publicados entre los años 2005-2013.

\section{Artículos publicados}

Como puede observarse en el Gráfico 4, la revista que más artículos ha publicado es Derecho, de la Pontificia Universidad Católica del Perú, con un total de 206 contribuciones. Le sigue Investigación Psicológica con 179 y Biblios en tercera posición con 141. Luego con 135 se encuentra otra revista de psicología, Liberabit, de la Universidad San Martín de Porres, junto con Arqueología y Sociedad de la Universidad de San Marcos. Seguidamente vemos tres revistas que superan los 100 artículos en el periodo: Boletín de Arqueología con 119, Cuadernos de Difusión, de Esan, que en 2009 cambia de nombre por Journal of Economics y alcanza los 118, y la Revista de Derecho de la Universidad de Piura con 110 e Investigación Educativa de la Universidad Nacional Mayor de San Marcos con un total de 109. Seguidamente vemos tres revistas que superan los 100 artículos en el periodo: Cuadernos de Difusión, de Esan, que en 2009 cambia de nombre por Journal of Economics y alcanza los 118, la Revista de Derecho de la Universidad de Piura con 110 e Investigación Educativa de la Universidad Nacional Mayor de San Marcos con un total de 109. En un grupo más compacto localizamos 11 revistas con una productividad que varía entre 70 y 90 artículos en los ocho años. Finalmente las que menos artículos han publicado son Debates de Sociología con 42 e Histórica con 57, ambas de la Universidad Católica. En el Gráfico 5 puede observarse la evolución de todas las publicaciones durante el intervalo analizado.

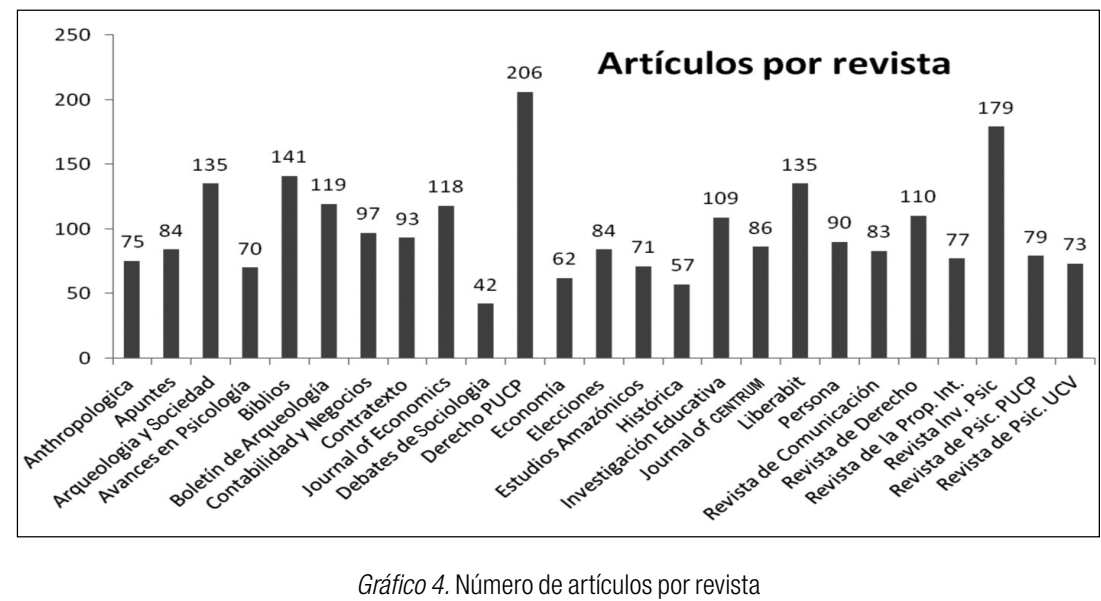




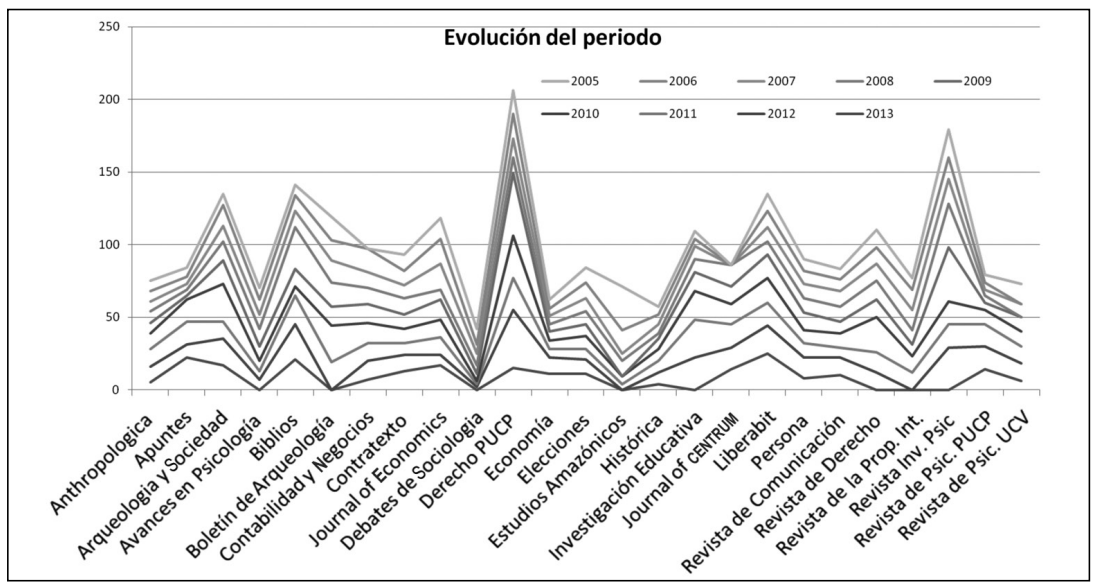

Gráfico 5. Evolución comparada

\section{Autores nacionales}

Hay un total de 3262 autores, de los cuales 1789 (54.2\%) son nacionales y 1473 (45.8 \%) extranjeros. La revista con más autores nacionales es Investigación en Psicología con 363, una media de 45.3 por año. Superan los 100 Arqueología y Sociedad e Investigación Educativa, ambas con 135, y Derecho con 114. Las que registran menos autores peruanos son Antrophologica con 24, seguida de Histórica con 21 y Comunicación y Biblios, con 20 cada una. Observamos como varía este indicador en dos revistas del mismo campo. Mientras Boletín de Arqueología tiene un $38.7 \%$ de autores nacionales, en Arqueología y Sociedad este porcentaje se eleva hasta el $70.3 \%$. En el caso de Derecho sucede algo parecido, la cuota de autoría nacional es del $64 \%$. En Debates de Sociología la proporción alcanza el $75 \%$, pero solamente con 33 artículos. En esta variable se produce en apariencia un fenómeno de concentración de autores nacionales basado no sólo en la proyección habitual de la revista, sino posiblemente porque el área temática no se presta a la acogida de contribuciones internacionales. Esto es especialmante notorio en revistas como Investigación Educativa o Derecho, que publican trabajos centrados en las problemáticas y la legislación nacional peruana. Debemos mencionar también que en ambas revistas se aprecia un elevado porcentaje de artículos producidos por profesores de la propia facultad. Contrariamente a esta tendencia, las revistas de ADE-Economía Journal of Economics y J. of CENTRUM Cathedra presentan un volumen bastante inferior de autores nacionales ( $\mathrm{Ta}$ bla3). 


\section{Autores extranjeros}

Contabilizamos un total de 1473 autores firmantes extranjeros. El número más elevado del total lo posee Biblios con 170 (89.5\%), seguido de J. of CENTRUM Cathedra con 132 (82.5\%). En un segundo grupo tenemos la Revista de Psicología de la Pontificia Universidad Católica del Perú con 121 (66.5 \%), el Boletín de Arqueología con 106 (61.3 \%) y Liberabit con 83 (61.1 \%) del total. Avances en Psicología, de la Universidad Femenina, registra sólo 23 autores extranjeros, que son el 21.7 \% del total de sus autores en los ocho años. La Revista de Psicología de la Universidad César Vallejo es un tanto más productiva en este aspecto, con 35 autores del exterior que alcanzan un $33.4 \%$. Si bien es una cifra pequeña, refleja un esfuerzo de internacionalización, tomando en cuenta que está editada por una universidad de reciente creación. Las revistas que menos autores extranjeros tienen son Debates de Sociología e Investigación Educativa, con 11 cada una. Estudios Amazónicos es la única publicación que no incluye ningún autor extranjero. La más equilibrada es Contabilidad y Negocios con 69 autores nacionales y 68 extranjeros. En total 11 de las 25 revistas analizadas tienen un mayor número de autores extranjeros que nacionales (Tabla 3 ).

\section{Internacionalidad de las contribuciones}

Otro indicador relevante asociado a la calidad de la publicación es el volumen de artículos en los que participan simultáneamente autores nacionales y extranjeros. La que mejor refleja esta forma de coautoría es el Boletín de Arqueología, con 18 artículos; siguen Arqueología y Sociedad con 7, la Revista de Psicología de la PUCP con 6, Liberabit con sólo 4, Antbropologica, Contabilidad y Negocios, Contratexto, J. of Economics e Histórica con 1. Trece revistas no reportan ningún artículo con estas características. En total hemos hallado 48 artículos con autores nacionales y extranjeros. En cifras porcentuales los autores nacionales llegan a representar el $54.2 \%$ del total, mientras que los extranjeros son el $45.8 \%$, lo cual muestra un nivel casi equiparado entre ambos tipos de autoría (Tabla 3).

Tabla 3. Producción por autores

\begin{tabular}{|l|c|c|c|c|c|}
\hline \multicolumn{1}{|c|}{ Revista } & AN & $\%$ & A EX & $\%$ & AN + AEX \\
\hline Anthropologica & 24 & 34.2 & 46 & 75.8 & 1 \\
\hline Apuntes & 60 & 60 & 40 & 40 & 3 \\
\hline Arqueología y Sociedad & 135 & 70.3 & 57 & 29.7 & 7 \\
\hline Avances en Psicología & 47 & 78.3 & 23 & 21.7 & 0 \\
\hline
\end{tabular}




\begin{tabular}{|l|c|c|c|c|c|}
\hline Biblios & 20 & 10.5 & 170 & 89.5 & 0 \\
\hline Boletín de Arqueología & 67 & 38.7 & 106 & 61.3 & 18 \\
\hline Contabilidad y Negocios & 69 & 50.3 & 68 & 49.7 & 1 \\
\hline Contratexto & 43 & 41.7 & 60 & 58.3 & 1 \\
\hline CD J.of Eonomics & 55 & 43.3 & 72 & 56.7 & 1 \\
\hline Debates de Sociología & 33 & 75 & 11 & 25 & 0 \\
\hline Derecho PUCP & 114 & 64 & 64 & 36 & 3 \\
\hline Economía & 47 & 69.1 & 21 & 30.9 & 0 \\
\hline Elecciones & 27 & 31.7 & 58 & 68.3 & 0 \\
\hline Estudios Amazónicos & 71 & 100 & 0 & 0 & 0 \\
\hline Histórica & 21 & 36.8 & 36 & 63.2 & 1 \\
\hline Investigación educativa & 135 & 92.4 & 11 & 7.6 & 0 \\
\hline J. of CENTRUM Cathedra & 28 & 17.5 & 132 & 82.5 & 0 \\
\hline Liberabit & 53 & 38.9 & 83 & 61.1 & 4 \\
\hline Persona & 50 & 44.6 & 62 & 55.4 & 2 \\
\hline Comunicación & 20 & 24 & 63 & 76 & 0 \\
\hline Revista de Derecho & 58 & 53.2 & 51 & 46.8 & 0 \\
\hline Revista de la Prop. Int. & 79 & 81.4 & 17 & 18.6 & 0 \\
\hline Inv. en Psicología & 363 & 84.6 & 66 & 15.4 & 0 \\
\hline Revista de Psic. PUCP & 61 & 33.5 & 121 & 66.5 & 6 \\
\hline Revista de Psic. UCV & 70 & 66.6 & 35 & 33.4 & 0 \\
\hline Total Revistas: 25 & 1789 & 54.2 & 1473 & 45.8 & 48 \\
\hline
\end{tabular}

\section{Autoría múltiple}

Veamos en este apartado las cifras de autoría entre dos o más autores y entre autores de diferentes países. El volumen de artículos por parejas de autores llega a los 338, Biblios es la que tiene el mayor volumen de autorías por parejas con 48 artículos; le sigue J. of CENTRUM Cathedra con 27, Arqueología y Sociedad con 22, Liberabit con 21 y Persona con 20. De tres autores Biblios es la que tiene el mayor número con 19, seguida de Liberabit con 17, Revista de Psicología de la PUCP y Persona con 12, e Investigación en Psicología con 10. Finalmente, Investigación en Psicología es la más flexible en número de contribuciones grupales con 45 artículos de cuatro o más autores, tiene incluso un artículo firmado por doce autores peruanos y 2 de once; Liberabit tiene 17, 2 de ellos con seis autores; Boletín de Arqueología tiene 11, 1 de diez y 2 de nueve autores. Once revistas no tienen ningún artículo de cuatro o más autores.

Respecto de la autoría entre investigadores de dos países, el Boletín de Arqueología tiene 16 contribuciones de autores de Perú/Estados Unidos, Perú/Japón, Perú/Reino Unido, Perú/Alemania. J. of CENTRUM Cathedra tiene 8, entre ellos Perú/España, Perú/Estados Unidos, India/Malasia, Estados 
Unidos/Nueva Zelanda. Liberabit tiene 6 más centradas en autores latinoamericanos como Argentina/Perú, Colombia/Chile. Por último, la Revista de Psicología de la PUCP cuenta con 4.

En los artículos con autores de tres o más países tenemos al Boletín de Arqueología con 2 y J. of CENTRUM Cathedra con 2. Finalmente, Arqueología y Sociedad y Contabilidad y Negocios con 1 . Veinte revistas no reportan ningún artículo escrito por autores de tres o más países.

En la Tabla 4 se resumen los datos de autorías múltiples.

Tabla 4. Producción científica autoría múltiple

\begin{tabular}{|l|c|c|c|c|c|}
\hline \multicolumn{1}{|c|}{ Revista } & $2 \mathrm{~A}$ & $3 \mathrm{~A}$ & $4+\mathrm{A}$ & $2 \mathrm{P}$ & $3+\mathrm{P}$ \\
\hline Anthropologica & 5 & 0 & 0 & 4 & 0 \\
\hline Apuntes & 17 & 3 & 1 & 6 & 0 \\
\hline Arqueología y Sociedad & 22 & 8 & 7 & 7 & 1 \\
\hline Avances en Psicología & 10 & 3 & 4 & 1 & 0 \\
\hline Biblios & 48 & 19 & 9 & 0 & 0 \\
\hline Boletín de Arqueología & 8 & 2 & 11 & 16 & 2 \\
\hline Contabilidad y Negocios & 8 & 8 & 0 & 2 & 1 \\
\hline Contratexto & 8 & 1 & 1 & 3 & 0 \\
\hline CD J.ofEconomics & 19 & 7 & 5 & 6 & 0 \\
\hline Debates de Sociología & 1 & 0 & 0 & 1 & 0 \\
\hline Derecho PUCP & 15 & 3 & 0 & 4 & 0 \\
\hline Economía & 14 & 0 & 0 & 1 & 0 \\
\hline Elecciones & 8 & 0 & 0 & 2 & 0 \\
\hline Estudios Amazónicos & 3 & 0 & 0 & 0 & 0 \\
\hline Histórica & 3 & 0 & 0 & 0 & 0 \\
\hline Investigación Educativa & 0 & 0 & 0 & 0 & 0 \\
\hline J. of CENTRUM Cathedra & 27 & 8 & 7 & 8 & 2 \\
\hline Liberabit & 21 & 17 & 17 & 6 & 0 \\
\hline Persona & 20 & 12 & 3 & 3 & 0 \\
\hline Comunicación & 13 & 5 & 3 & 0 & 0 \\
\hline Revista de Derecho & 2 & 0 & 0 & 0 & 0 \\
\hline Revista de la Prop. Int. & 13 & 4 & 0 & 1 & 0 \\
\hline Inv. en Psicología & 19 & 10 & 45 & 0 & 0 \\
\hline Revista de Psic. PUCP & 16 & 12 & 12 & 4 & 1 \\
\hline Revista de Psic. UCV & 18 & 7 & 8 & 1 & 0 \\
\hline \multicolumn{1}{|c|}{ Total Revistas: 25 } & 338 & 129 & 133 & 76 & 8 \\
\hline & & & & & \\
\hline
\end{tabular}

\section{Campo 3. Indicadores de calidad}

En esta sección analizamos las citas a los artículos de las revistas producidas durante el periodo de análisis. Empleamos dos indicadores de visibilidad de 
citas: Google Académico y citas en el buscador Clase: Citas Latinoamericanas en Ciencias Sociales y Humanidades (Tabla 5 ).

La revista con mayor número de citas en Google Académico es Revista Investigación Psicológica de la Universidad de San Marcos, con 404 citas. Es de hecho la que tiene mayores referencias con distintos artículos citados, entre ellos Martínez, M. 2006. "La investigación cualitativa: síntesis conceptual”, 9 (1), 123-146, con 154, o Riveros, Q., V. Hernández y B. Rivera. 2007. "Niveles de depresión y ansiedad en estudiantes universitarios de Lima metropolitana", 10 (1), 91-102, con 39. Vemos que la revista J. of CENTRUM Cathedra también está muy citada en este buscador, registra 149 referencias. El artículo mejor posicionado es Zhu, J. 2011. "Airlines performance via two-stage network DEA approach”, 4 (2), 260-269, con 13 citas, seguido de Sumner, S., R. Johnsony L. Soenen. 2010. "Spillover effects among gold, stocks, and bonds", 3 (2), 106-120, con 12. En tercer lugar tenemos a la Revista de Psicología de la PUCP con 100 citas, el artículo más citado es Zubieta, E., M. Beramendi, F. Sosa y J. A. Torres. 2011. "Sexismo ambivalente, estereotipos y valores en el ámbito militar”, 29 (1), 101-130, con 5 citas. Destaca también Biblios con dos artículos de 23 citas cada uno.

Datos diferentes se aprecian en las citas que almacena Clase. Podemos apreciar que no existe una correlación con los datos de Google Académico. La revista citada con mayor frecuencia es Histórica, que registra 431 citas; le sigue Economía con 139, Boletín de Arqueología con 136 y muy cerca con 134 Debates de Sociología, que en Google Académico sólo tiene 2 entradas registradas.

En cuanto a los criterios Latindex, sólo Apuntes cumple con el total de 33. Con 32 se encuentran Biblios, Contabilidad y Negocios, Derecho (PUCP), Liberabit, Persona, Revista de Comunicación y Revista de Psicología (PUCP). Las evaluadas con puntaje más bajo son Estudios Amazónicos y Revista de la Competencia Intelectual, con tan sólo 25 criterios cumplidos. Histórica, que alcanza los 26 criterios cumplidos, muestra 431 citas en Clase, en tanto que Economía también cumple con 26 criterios Latindex y alcanza 139 entradas en Clase.

Tabla 5. Citaciones y Criterios Latindex

\begin{tabular}{|l|c|c|c|}
\hline \multicolumn{1}{|c|}{ Revista } & GA & CLASE & C.LI. \\
\hline Anthropologica & 34 & 0 & 31 \\
\hline Apuntes & 23 & 0 & 33 \\
\hline Arqueología y Sociedad & 17 & 0 & 26 \\
\hline Avances en Psicología & 1 & 0 & 29 \\
\hline Biblios & 46 & 0 & 32 \\
\hline Boletín de Arqueología & 43 & 136 & 29 \\
\hline Contabilidad y Negocios & 1 & 63 & 32 \\
\hline Contratexto & 20 & 0 & 30 \\
\hline
\end{tabular}




\begin{tabular}{|l|c|c|c|}
\hline Journal of Economics & 9 & 0 & 27 \\
\hline Debates de Sociología & 2 & 134 & 29 \\
\hline Derecho PUCP & 29 & 111 & 32 \\
\hline Economía & 2 & 139 & 26 \\
\hline Elecciones & 0 & 0 & 30 \\
\hline Estudios Amazónicos & 0 & 0 & 25 \\
\hline Histórica & 4 & 431 & 26 \\
\hline Investigación Educativa & 0 & 21 & 31 \\
\hline J. of CENTRUM Cathedra & 149 & 0 & 29 \\
\hline Liberabit & 9 & 99 & 32 \\
\hline Persona & 14 & 0 & 32 \\
\hline Revista de Comunicación & 15 & 0 & 32 \\
\hline Revista de Derecho & 0 & 0 & 31 \\
\hline Revista Comp. intelectual & 14 & 0 & 25 \\
\hline Revista Inv. Psicológica & 404 & 0 & 30 \\
\hline R.de Psicología PUCP & 100 & 0 & 32 \\
\hline R. de Psicología UCV & 6 & 0 & 31 \\
\hline
\end{tabular}

\section{Indexación en otros buscadores y sistema Open Access}

Por último, veamos la visibilidad de las revistas en otros buscadores (Tabla 6). Once revistas son visibles en Dialnet, 3 en Scielo y 4 en Redalyc. Quince emplean el sistema de edición Open Access (OA). Todas las revistas de la Pontificia Universidad Católica del Perú se encuentran reunidas en el Portal de Revistas PUCP, mientras que las de la Universidad de San Marcos son accesibles mediante el Sistema Digital de Bibliotecas.

Tabla 6. Indexación en otros buscadores

\begin{tabular}{|l|c|c|c|c|}
\hline & Dialnet & Scielo & Redalyc & $\mathrm{OA}$ \\
\hline Anthropologica & $\mathrm{X}$ & $\mathrm{X}$ & & $\mathrm{X}$ \\
\hline Apuntes & & & & \\
\hline Arqueología y Sociedad & & & & \\
\hline Avances en Psicología & & & & \\
\hline Biblios & $\mathrm{X}$ & & $\mathrm{X}$ & $\mathrm{X}$ \\
\hline Boletín de Arqueología & $\mathrm{X}$ & & & $\mathrm{X}$ \\
\hline Contabilidad y Negocios & & & $\mathrm{X}$ & $\mathrm{X}$ \\
\hline Contratexto & $\mathrm{X}$ & & & \\
\hline Journal of Economics & & $\mathrm{X}$ & & $\mathrm{X}$ \\
\hline Debates de Sociología & & & & $\mathrm{X}$ \\
\hline Derecho PUCP & & & & $\mathrm{X}$ \\
\hline Economía & & & & $\mathrm{X}$ \\
\hline Elecciones & $\mathrm{X}$ & & & \\
\hline Estudios Amazónicos & & & & \\
\hline
\end{tabular}




\begin{tabular}{|l|c|c|c|c|}
\hline Histórica & $\mathrm{X}$ & & & $\mathrm{X}$ \\
\hline Investigación Educativa & & & & $\mathrm{X}$ \\
\hline J. of CENTRUM Cathedra & & & & $\mathrm{X}$ \\
\hline Liberabit & $\mathrm{X}$ & & $\mathrm{X}$ & $\mathrm{X}$ \\
\hline Persona & $\mathrm{X}$ & & $\mathrm{X}$ & $\mathrm{X}$ \\
\hline Revista de Comunicación & $\mathrm{X}$ & & & \\
\hline Revista de Derecho & & & & \\
\hline Revista Comp. Intelectual & & & & \\
\hline Revista Inv. Psic & $\mathrm{X}$ & & & $\mathrm{X}$ \\
\hline Revista de Psic. PUCP & $\mathrm{X}$ & $\mathrm{X}$ & & $\mathrm{X}$ \\
\hline Revista de Psic. UCV & & & & \\
\hline & 11 & 3 & 4 & 16 \\
\hline
\end{tabular}

\section{Discusión}

En cuanto a la metodología de trabajo, creemos que el sistema de recolección de datos que hemos empleado para esta investigación ha funcionado de manera satisfactoria para obtener los datos respecto de las distintas variables que nos hemos propuesto analizar. Ha sido una tarea larga y compleja, debido a que la localización de los datos correspondientes a las variables de cada una de las revistas indexadas se ha introducido de forma manual e individualizada en el fichero de datos. El hecho de que no se disponga de una herramienta que pueda filtrar las búsquedas y ofrecer resultados listados de forma automática puede haber provocado algunos errores humanos en el ingreso y cuantificación de los datos por posibles errores mecánicos. No obstante, este procedimiento era el único posible para poder obtener el mapa de la producción científica que nos proponíamos en tanto que no existen instrumentos disponibles para realizarlo.

Resultaría interesante que Latindex incorpore en su sistema información más precisa para los usuarios, por ejemplo, qué criterios se han cumplido satisfactoriamente durante la evaluación de la publicación, sistemas de filtros más precisos en la opción "Catálogo", poder disponer de herramientas adicionales para buscar citas y versiones de un mismo artículo o hacer búsquedas específicas por revista, institución y autor. Finalmente, integrar la plataforma con otros buscadores similares como Clase o Scielo sería de gran ayuda para continuar provechosamente en esta línea.

El análisis de las cifras permite realizar interpretaciones diferentes, según cómo abordemos los datos. En líneas generales hemos podido constatar una producción significativa de artículos que alcanza los 2 475, equivalente a una 
media de 100 artículos por revista para el periodo de ocho años analizado. Sin embargo, no podemos decir que la producción y volumen de citaciones es homogénea entre todas ellas, pese a que se observan esfuerzos institucionales, un tanto aislados, para intentar cubrir y proyectar socialmente unas preferencias temáticas o simplemente dar a conocer la actividad investigadora de sus académicos. Esto queda ejemplificado en el trabajo de la Universidad Católica, cuyas nueve revistas se publican regularmente. Incluso todas ellas mantienen un estilo corporativo de diseño de portales, revista y están disponibles tanto en papel como en web abierta. Sin embargo, las diferencias entre ellas comienzan a advertirse cuando examinamos el volumen y variedad de artículos que ofrecen. Aun cuando éste ya es un tema que escapa los límites fijados por este artículo, podríamos decir que parece suceder el mismo fenómeno que en otras publicaciones, cuyo indicador de potencia queda fijado por el nivel de los investigadores que participan en ella, por las facilidades y contactos que puede suministrar el departamento que la gestiona y en general su dimensión corporativa. Esto hace que, en términos prácticos, el resultado final sea como verificamos: más o menos artículos, mayor o menor presencia de autores internacionales previamente referenciados, citas, etc. Hay algunas revistas que incluso han dejado de publicarse en los dos últimos años, si bien las interrupciones no tienen necesariamente una relación con el nivel de citas.

El segundo caso a destacar es el de la Universidad de San Marcos, universidad pública que en medio de las dificultades presupuestales ha podido posicionarse con tres revistas que contienen un total de 423 artículos. Investigación Psicológica es la publicación que tiene más citas en el Google Académico, con 404. El resto de universidades editoras son privadas y no gestionan más de dos revistas cada una; entre ellas se encuentran la Universidad de Lima y la Universidad de Piura. En estos casos ya se trata de iniciativas más aisladas que evidencian un interés reducido por participar en el circuito universitario o porque sencillamente les resulta poco viable poder lograr la edición de los números y disponer de los recursos logísticos necesarios para poner en ejecución un proceso de calidad editorial riguroso: disponer de un comité editorial, pares ciegos, etc.

Mención especial merece el trabajo que ha realizado Biblios, revista de biblioteconomía gestionada por una empresa privada. Esta publicación muestra algunos de los mejores indicadores generales y posee el mayor número de artículos de autores extranjeros (170) y el mayor volumen de artículos de dos autores (48). Tiene 46 citas internacionales.

Si efectuamos una revisión comparativa por áreas temáticas (Tabla 7), las revistas del ámbito de Arqueología, Antropología e Historia son las mejor 
citadas (665), aun cuando sean las terceras en volumen de artículos publicados y contabilicen un total de 5 publicaciones. Las de Psicología ocupan el segundo lugar con 6 publicaciones, 626 artículos y 633 citas. ADE-Economía presenta datos muy relevantes con 5 revistas y alcanza las 386 citas. Más relegadas están las de Derecho, con 4 revistas, 477 artículos y 154 citas. Con menos de 50 citas tenemos Comunicación, Sociología, Educación y Biblioteconomía. Esta última es una sola revista que no muestra citas.

Tabla 7. Tabla comparada de citaciones

\begin{tabular}{|c|l|c|c|c|c|c|}
\hline & & $\mathbf{N}^{0} \mathbf{R}$ & Arts. & AN & AEXT & Citas \\
\hline 1 & Arq/Antr/Hist & 5 & 457 & 318 & 245 & 665 \\
\hline 2 & Psicología & 6 & 626 & 644 & 390 & 633 \\
\hline 3 & ADE-Economía & 5 & 447 & 259 & 333 & 386 \\
\hline 4 & Derecho & 4 & 477 & 317 & 190 & 154 \\
\hline 5 & Biblioteconomía & 1 & 141 & 20 & 170 & 46 \\
\hline 6 & Comunicación & 2 & 176 & 63 & 123 & 35 \\
\hline 7 & Sociología & 1 & 42 & 33 & 11 & 2 \\
\hline 8 & Educación & 1 & 109 & 135 & 11 & 0 \\
\hline
\end{tabular}

\section{CONCLUSIONES Y RECOMENDACIONES}

Los resultados de este estudio indican que hay un mayor volumen de revistas y autores afiliados a universidades privadas respecto de universidades públicas. En total existen 18 revistas adscritas a universidades privadas, la mitad de ellas de la Pontificia Universidad Católica del Perú: Anthropologica, Boletín de Arqueología, Contabilidad y Negocios, Debates de Sociología, Derecho, Economía, Histórica, J. of CENTRUM Cathedra y Revista de Psicología. La única universidad pública que figura en el inventario es la Universidad Nacional Mayor de San Marcos, que edita 3 revistas: Arqueología y Sociedad, Investigación Educativa e Investigación en Psicología. El resto de centros edita sólo una o dos revistas.

A partir del análisis de los datos podemos afirmar que existe un crecimiento significativo de la producción editorial en formato revista, centrado principalmente en las iniciativas propias de los centros universitarios privados en detrimento de los públicos. Esto se constata especialmente por un incremento del número de artículos, autores, internacionalidad de las contribuciones, un mayor rigor en el cumplimiento de las normas editoriales y procesos de revisión por pares, así como de visibilidad en buscadores internacionales.

Este estudio ha permitido constatar la irregularidad de algunas revistas. Hemos localizado varias con aparición intensa pero episódica, razones que 
nos han llevado a descartarlas de la muestra definitiva. Situación similar sucede con las de reciente aparición.

Los resultados son insuficientes para poder vincular directamente la producción científica de la revista con grupos de investigación, líneas concretas o relaciones con otras instituciones. Quizás donde se aprecia más nítidamente este aspecto es en las revistas del ámbito de la biblioteconomía, economía y la arqueología, donde el volumen de autores afiliados a centros e instituciones nacionales e internacionales es bastante elevado y variado en cuanto a temáticas y países, reproduciendo el modelo de coautoría de revistas de primer nivel.

Una tarea pendiente de los editores es impulsar medidas para aumentar la visibilidad de las revistas en el ámbito internacional. Aún son muy pocas las revistas indexadas en otros catálogos regionales como Clase o Redalyc. Ninguna figura en SJR Scopus ni en ISI. Las revistas de la Pontificia Universidad Católica del Perú son las que evidencian un mayor cuidado en cuanto a los estándares de calidad y son gestionadas conjuntamente mediante un servicio de publicaciones. La Universidad de San Marcos posee una plataforma de gestión propia, similar al buscador Scielo-Perú, pero limitada en sus opciones de localización de artículos. La web Amauta que representa el repositorio nacional no facilita la ubicación de artículos, no ofrece índices de impacto para las revistas ni citas por autores. Se ha de incidir en la formación de bibliotecarios para mejorar la tecnificación y navegabilidad de las plataformas existentes y optar conjuntamente por el modelo de Open Access. Asimismo, es necesario tener presencia en buscadores internacionales y optimizar los procesos de revisión y publicación para incentivar la participación de autores nacionales y sobre todo extranjeros. Como ya han advertido algunos autores del campo de la medicina (Huamaní y Pacheco Romero, 2009; Cabezas y Mayta-Tristán, 2008) mediante el cumplimento de estas medidas se podrá obtener un nivel de presencia internacional significativo, al menos en el ámbito iberoamericano.

Finalmente, aunque parezcan sencillas, implementar cada una de estas acciones requiere de presupuesto, conocimientos específicos y capacitación de personas. Esta es una buena base para poder comprender cómo la mejora de la calidad de las publicaciones es finalmente un valor añadido para autores, programas y centros. Esto sin lugar a dudas es un fenómeno complejo de gestionar, que debe ser enfrentado seriamente por el Estado y las propias instituciones universitarias que son las encargadas de marcar las políticas. Sólo si se implementan acciones serias y estables en el tiempo podrán conducirnos a resultados positivos en los próximos años. 


\section{REFERENCIAS}

Alarcón-Villaverde, J., F. Romaní y C. Gutiérrez. 2010. “Publicaciones científicas estudiantiles producidas en el curso de Epidemiología de la Facultad de Medicina de la Universidad Nacional Mayor de San Marcos durante el periodo 2003-2009". Anales de la Facultad de Medicina 71 (2) (abril): 111-116. UNMSM. Facultad de Medicina.

Alarcon, J., J. Murillo, J. Piscoya, C. Castro y C. Isasi. 1996. "Evolución y características de las publicaciones biomédicas peruanas, 1985-1993". Anales Facultad Medicina (Perú) 57 (3): 158-173.

Alarcón, R. 1980. "Desarrollo y estado actual de la psicología en el Perú (Parte C)”. Revista latinoamericana de Psicología 12 (2): 225 235.

Alcain, María Dolores, Adelaida Román Román y Elea Giménez Toledo. 2008. "Categorización de las revistas españolas de Ciencias Sociales y Humanas en RESH”. Revista Española de Documentación Cientifica 31 (1) (enero-marzo): 85-95.

__ y M. Luisa Lascurain. 2002. "Análisis de las revistas latinoamericanas de psicología incluidas en el "Directorio Latindex". Papeles del Psicólogo 83: 34-43.

Arroyo-Hernández, C. H., E. B. Zukerán-Medina y U. E. Miranda-Soberón. 2009. "Características de la producción científica biomédica en Ica, Perú 1998-2007”. Revista Peruana de Medicina Experimental y Salud Pública 26 (2): 203-206.

Borrego, Ángel y Cristóbal Urbano. 2006. "La evaluación de revistas científicas en Ciencias Sociales y Humanidades". Información, cultura y sociedad 14 (enero-junio): 11-27. Ciudad Autónoma de Buenos Aires.

Burstein, Z. y C. Cabezas. 2010. "Advances and challenges of the Revista Peruana de Medicina Experimental y Salud Pública in the year 2010". Revista Peruana de Medicina Experimental y Salud Pública 27 (1): 3-5.

Cabezas, César y Percy Mayta-Tristán. 2008. "Evolución y perspectivas de la Revista Peruana de Medicina Experimental y Salud Pública, 1945-2008”. Revista Peruana de Medicina Experimental y Salud Pública 25 (2) (abril-junio): 167-168. ISSN 1726-4634.

Cáceres, C. F. y W. Mendoza. 2009. "Globalized research and "national science": the case of Peru". American Journal of Public Health 99 (10): 1792-1798.

Cuevas, R. F. y M. Mestaza. 2002. "La evaluación científica y el sistema de revisión por pares". CSI Boletín 46: 1-5.

Fernández-Quijada, D. 2010. "El perfil de las revistas españolas de comunicación (2007-2008)”. Revista española de documentación cientifica 33 (4): 553-581.

_ 2009. "Revistas científicas e índices de impacto: A propósito de "Hacer saber". Área abierta 20: 1-10. 
Gamboa, J. O. 1998. "Acceso a revistas latinoamericanas en Internet: opción a través de las bases de datos Clase y Periódica". Ciência da informação 27 (1), 0-0.

Gerstein, N. B. 2005. Calidad de las revistas cientificas biomédicas peruanas. Academia Nacional de Medicina del Perú. Disponible en: http://www.acadnacmedicina.org.pe/publicaciones/anal_agodic04 /03sesordinaria/02Naldo_Balarezo.pdf

Gutiérrez, C. y P. Mayta-Tristán. 2003. "Publicación desde el pregrado en Latinoamérica: importancia, limitaciones y alternativas de solución". Cimel 8 (1): 54-60.

Huamaní, C. y J. Pacheco-Romero. 2011. "Colaboración científica en artículos de revistas biomédicas peruanas”. Anales de la Facultad de Medicina 72 (4) (diciembre): 261-268. UNMSM. Facultad de Medicina.

Huamaní, C. y P. Mayta-Tristán. 2008. "Factor de impacto modificado de las revistas médicas indizadas en SciELO Perú, 2006”. Revista Peruana de Medicina Experimental y Salud Pública 25 (3): 349350.

Huamaní, C. y J. Pacheco-Romero. 2009. "Visibilidad y producción de las revistas biomédicas peruanas". Revista de Gastroenterología del Perú 29 (2): 132-139.

Huamaní, C., P. Chávez-Solís y P. Mayta-Tristán. 2008. “Aporte estudiantil en la publicación de artículos científicos en revistas médicas indizadas en Scielo-Perú, 1997-2005”. Anales de la Facultad de Medicina 69 (1): 42-45. UNMSM. Facultad de Medicina.

Huamaní, C. y P. Mayta-Tristán. 2010. "Producción científica peruana en medicina y redes de colaboración, análisis del Science Citation Index 2000-2009”. Revista Peruana de Medicina Experimental y Salud Pública 27 (3): 315-325.

López Baena, A. J., M. Valcárcel Cases y M. Barbancho Medina. 2005. "Propuesta de un sistema de evaluación de revistas científica en las áreas de Ciencias Humanas y Sociales". Revista española de documentación científica 28 (1): 22-48.

Lluch Osca, Julia y María Elena Mateo Marquina. 2003. “Difusión de las revistas españolas de ciencias sociales y humanidades. Acercamiento bibliométrico". Revista General de Información y Documentación 13 (1): 115-132.

Mayta-Tristán, Percy y Américo Pena-Oscuvilca. 2009. "Importancia de la publicación en las sociedades científicas de estudiantes de medicina del Perú: estudio preliminar". CIMEL 14 (1): 27-34. ISSN 1680-8398.

Miguel, S. 2011. "Revistas y producción científica de América Latina y el Caribe: su visibilidad en SciELO, RedALyC y SCOPUS”. Revista Interamericana de Bibliotecología 34 (2): 187-199. 
Miguel Villamón-Herrera, José Devís-Devís, Alexandra Valencia-Peris y Javier Valenciano-Valcárcel. 2007. "Características y difusión de las revistas científico-técnicas españolas de ciencias de la actividad física y el deporte”. El Profesional de la Información 16 (6) (noviembre-diciembre): 605-615.

Miyahira Arakaki, J. M. 2008. "Criterios de calidad de las revistas científicas”. Revista Médica Herediana 19 (1): 01-04.

Molina-Ordóñez, J., C. Huamaní y P. Mayta-Tristán, P. 2008. “Apreciación estudiantil sobre la capacitación universitaria en investigación: estudio preliminar". Revista Peruana de Medicina Experimentaly Salud Pública 25 (3): 325-329.

Pamo Reyna, O. G. 2005. "Estado actual de las publicaciones periódicas científicas médicas del Perú”. Revista Médica Herediana 16 (1): 65-73.

Román Román, Adelaida, Manuela Vázquez Valero y Carmen Urdín Camino. 2003. "Las Revistas Españolas de Ciencias de la salud frente a los criterios de calidad editorial Latindex". Revista Española de Documentación Científica 26 (4): 418-432.

_ Manuela Vázquez Valero y Carmen Urdín Camino. 2002. "Los criterios de calidad editorial Latindex en el marco de la evaluación de las revistas españolas de humanidades y ciencias sociales". Revista Española de Documentación Científica 25 (3): 286-307.

Romaní, F. y P. Wong. 2009. "Reporte y serie de casos en el Perú: situación de un tipo de publicación subestimada. Análisis de las revistas médicas peruanas indizadas en SciELO-Perú, 1997-2008”. Revista Perú Epidemiology 13 (3): 1-7.

Taype-Rondán, Á., Y. Lajo-Aurazo, R. Gutiérrez-Brown, N. Zamalloa-Masías y M. Saldaña-Gonzales. 2011. "Aporte de las sociedades estudiantiles en la publicación científica en SciELO-Perú, 2009-2010". Revista Peruana de Medicina Experimental y Salud Pública 28 (4): 691-692.

Varela Pinedo, L., P. J. Ortiz Saavedra y H. Chávez Jimeno. 2003. "Características de los trabajos científicos nacionales en geriatría y gerontología. 1980-2001”. Revista Médica Herediana 14 (1): 18 25.

Vázquez Valero, M., C. Urdín Caminos y A. Román Román. 2003. "Las revistas españolas de ciencias de la salud frente a los criterios de calidad editorial LATINDEX". Revista española de documentación científica 26 (4): 56-73. 
Anexo 1

\begin{tabular}{|c|c|c|c|c|c|c|c|c|c|}
\hline Revista & 2013 & 2012 & 2011 & 2010 & 2009 & 2008 & 2007 & 2006 & 2005 \\
\hline $\begin{array}{l}\text { Boletín de la Academia } \\
\text { Peruana de la Lengua }\end{array}$ & 0 & 0 & 0 & 0 & 0 & 9 & 9 & 10 & 0 \\
\hline Comunicacción & 6 & 12 & 13 & 9 & 0 & 0 & 0 & 0 & 0 \\
\hline Comunifé & 0 & 0 & 0 & 8 & 0 & 8 & 9 & 7 & 11 \\
\hline Educación & 0 & 0 & 0 & 12 & 0 & 0 & 0 & 35 & 40 \\
\hline lus et veritas & \multicolumn{9}{|c|}{ No Disponible } \\
\hline La Tribuna del abogado & 0 & 219 & 166 & 24 & 0 & 0 & 0 & 0 & 0 \\
\hline Lex & 28 & 0 & 0 & 0 & 0 & 0 & 0 & 0 & 0 \\
\hline Nuevos paradigmas & \multirow{2}{*}{\multicolumn{9}{|c|}{ No Disponible }} \\
\hline Pacarina del sur & & & & & & & & & \\
\hline R. de la Comp. Intelectual & 0 & 0 & 12 & 11 & 8 & 10 & 14 & 14 & 8 \\
\hline R. Ingemmet & \multicolumn{9}{|c|}{ No es del área de ciencias sociales } \\
\hline $\begin{array}{l}\text { Revista de Psicología de la } \\
\text { Universidad Católica Santa } \\
\text { María }\end{array}$ & 0 & 0 & 0 & 0 & 7 & 7 & 6 & 6 & 5 \\
\hline $\begin{array}{l}\text { Revista de Psicología } \\
\text { (Arequipa) }\end{array}$ & 0 & 0 & 8 & 0 & 0 & 0 & 0 & 0 & 0 \\
\hline Revista Estrategias & 5 & 15 & 0 & 0 & 0 & 0 & 0 & 0 & 0 \\
\hline R. Juridica del IPEF & \multirow{3}{*}{\multicolumn{9}{|c|}{ No disponible }} \\
\hline $\begin{array}{l}\text { Revista Peruana de } \\
\text { Psicología y Trabajo Social }\end{array}$ & & & & & & & & & \\
\hline R. Psicológica Herediana & & & & & & & & & \\
\hline Tzhoecoen & 0 & 0 & 21 & 21 & 13 & 10 & 0 & 0 & 0 \\
\hline Zonas áridas & & & & & & & 22 & 15 & 8 \\
\hline
\end{tabular}

Para citar este artículo:

Morales Morante, Luís Fernando. 2016. "Producción e impacto de las revistas peruanas del ámbito de las Ciencias Sociales en el catálogo Latindex." Investigación Bibliotecológica: Archivonomía, Bibliotecología e Información 69: 179-204. http://dx.doi.org/10.1016/j.ib bai.2016.04.017 